\title{
The usefulness of analytic response functions ${ }^{\star}$
}

\author{
D. Orozco Suárez ${ }^{1}$ and J. C. del Toro Iniesta ${ }^{1}$
}

\begin{abstract}
Instituto de Astrofísica de Andalucía, Consejo Superior de Investigaciones Científicas, Apdo. de Correos 3004, 18080 Granada, Spain e-mail: orozco@iaa.es; jti@iaa.es
\end{abstract}

Received 7 August 2006 / Accepted 8 October 2006

\section{ABSTRACT}

\begin{abstract}
Aims. We introduce analytical response functions and their main properties as an important diagnostic tool that help understand Stokes profile formation physics and the meaning of well-known behaviors of standard inversion codes of the radiative transfer equation often used to measure solar magnetic fields.

Methods. A Milne-Eddington model atmosphere is used as an example where response functions are analytical. A sample spectral line has been chosen to show the main qualitative properties.

Results. We show that analytic response functions readily provide explanations for various well-known behaviors of spectral lines, such as the sensitivity of visible lines to weak magnetic fields or the trade-offs often detected in inversion codes between the MilneEddington thermodynamic parameters. We also show that response functions are helpful in selecting sample wavelengths optimized for specific parameter diagnostics.
\end{abstract}

Key words. radiative transfer - magnetic fields - line: formation - polarization - Sun: photosphere - Sun: magnetic fields

\section{Introduction}

Diagnosing the solar atmosphere from spectropolarimetric observations is one of the most important subjects of current solar physics. Both the theoretical understanding of the physical processes taking place in the photosphere and the design of new instrumentation that improve our ability to obtain more and better information from the Sun can be improved by a thorough study of the radiative transfer equation (hereafter referred to as RTE). RTE is the only tool available to describe the problem mathematically. Approximations have been devised according to the observational and the post-facto computational capabilities. The Milne-Eddington (M-E) approximation has provided insight into the physical processes taking place in line formation and inferring accurate values of several physical parameters of the solar atmosphere. Its specific analytical character is its most powerful feature.

A physical analysis of the sensitivities of spectral lines in terms of analytic mathematical functions is still missing in the literature and may provide a better understanding of how the solar parameters influence the shape of the observed Stokes profiles of these spectral lines and explanations for the tradeoffs and other well known behaviors of inversion codes currently used for the inference of such solar atmospheric parameters. Here we introduce the analytic response functions (RFs) of Stokes profiles as formed in M-E model atmospheres and discuss their main properties.

Weighting functions for unpolarized light (Mein 1971) were the precursors of RFs, extended to polarized light by Landi Degl'Innocenti \& Landi Degl'Innocenti (1977). As explained by Ruiz Cobo \& del Toro Iniesta (1994; see also del Toro Iniesta 2003), RFs provide the sensitivities of Stokes profiles to the various atmospheric quantities playing a role in

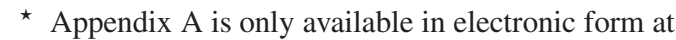
http : //www . aanda.org line formation. Since all these quantities are constant with depth in a M-E atmosphere, M-E RFs are simply partial derivatives of the analytic solution of the RTE with respect to the model parameters. This feature enables us to deduce analytic formulae for the sensitivities (they are explicitly written in the Appendix) and to study their characteristics and properties. Such properties are shown to be useful in practice in understanding the behavior of spectral lines as well as in helping in line and sample selection when designing new instruments.

\section{The response functions in a Milne Eddington atmosphere}

\subsection{Summary of radiative transfer}

The radiative transfer equation (RTE) for polarized light in a plane-parallel atmosphere is

$\frac{\mathrm{d} \boldsymbol{I}}{\mathrm{d} \tau}=\mathbf{K}(\boldsymbol{I}-\boldsymbol{S})$

where $\boldsymbol{I}=(I, Q, U, V)^{\mathrm{T}}$ stands for the Stokes vector which gives a full description of the polarization state of light, $\tau$ for the continuum optical depth at a reference wavelength, $\mathbf{K}$ for the $4 \times 4$ propagation matrix, $\boldsymbol{S}$ for the source function vector and ${ }^{\mathrm{T}}$ means the transpose. All the medium properties relevant to line formation are contained in $\mathbf{K}$ and $\boldsymbol{S}$. In LTE conditions, $\boldsymbol{S}=\left(B_{\lambda}(T), 0,0,0\right)^{\mathrm{T}}$, where $B_{\lambda}(T)$ is the Planck function at the local temperature $T$.

In a Milne-Eddington (M-E) model atmosphere, an analytical solution is found for the RTE (see, e.g. Unno 1956; Rachkovsky 1962, 1967; Landolfi \& Landi Degl'Innocenti 1982). In such an atmosphere, all the atmospheric quantities are constant with depth except for the source function that varies linearly:

$\boldsymbol{S}=\mathbf{S}_{0}+\mathbf{S}_{1} \tau=\left(S_{0}+S_{1} \tau\right)(1,0,0,0)^{\mathrm{T}}$. 
The propagation matrix is also constant with depth. Following, e.g., the notation in del Toro Iniesta (2003), such an analytical solution reads

$$
\begin{aligned}
I & =S_{0}+\Delta^{-1}\left[\eta_{I}\left(\eta_{I}^{2}+\rho_{Q}^{2}+\rho_{U}^{2}+\rho_{V}^{2}\right)\right] S_{1}, \\
Q & =-\Delta^{-1}\left[\eta_{I}^{2} \eta_{Q}+\eta_{I}\left(\eta_{V} \rho_{U}-\eta_{U} \rho_{V}\right)+\rho_{Q} \Pi\right] S_{1}, \\
U & =-\Delta^{-1}\left[\eta_{I}^{2} \eta_{U}+\eta_{I}\left(\eta_{Q} \rho_{V}-\eta_{V} \rho_{Q}\right)+\rho_{U} \Pi\right] S_{1}, \\
V & =-\Delta^{-1}\left[\eta_{I}^{2} \eta_{V}+\eta_{I}\left(\eta_{U} \rho_{Q}-\eta_{Q} \rho_{U}\right)+\rho_{V} \Pi\right] S_{1},
\end{aligned}
$$

with

$\Delta=\eta_{I}^{2}\left(\eta_{I}^{2}-\eta_{Q}^{2}-\eta_{U}^{2}-\eta_{V}^{2}+\rho_{Q}^{2}+\rho_{U}^{2}+\rho_{V}^{2}\right)-\Pi^{2}$,

where

$\Pi=\eta_{Q} \rho_{Q}+\eta_{U} \rho_{U}+\eta_{V} \rho_{V}$

It can easily be seen that $\eta_{I}, \eta_{Q}, \eta_{U}, \eta_{V}, \rho_{Q}, \rho_{U}$, and $\rho_{V}$, and hence the solution depends on just nine parameters, $(B, \gamma, \chi)$, the strength, inclination and azimuth of the magnetic field vector on the local reference frame, on $S_{0}, S_{1}$, the two parameters describing the source function, on $\eta_{0}$, the line-to-continuum absorption coefficient ratio, on $\Delta \lambda_{\mathrm{D}}$, the Doppler width of the line, on the damping parameter $a$, and on the line-of-sight velocity, $v_{\text {LOS. }}$.

\subsection{Milne-Eddington response functions}

According to Ruiz Cobo \& del Toro Iniesta (1994) (see also del Toro Iniesta \& Ruiz Cobo 1996; or del Toro Iniesta 2003) the sensitivity of the Stokes profiles to perturbations of the atmospheric physical quantities is given by the response functions (RFs). Fortunately, in the specific case of constant quantities (model parameters) with depth, as is the case of an M-E atmosphere, such RFs are the result of integrating in depth the regular RFs. Such $\tau$-integrated response functions are thus simply functions of the wavelength and can be considered as the partial derivatives of the Stokes vector with respect to the corresponding model parameter:

$\boldsymbol{R}_{x}(\lambda)=\frac{\partial \boldsymbol{I}(\lambda)}{\partial x}$,

where $x$ represents any of the model parameters. We hereafter refer to these $\tau$-integrated RFs as M-E RFs or just RFs.

Therefore, by taking derivatives of the analytical solution (3), the sensitivities of the Stokes profiles to perturbations of the M-E model parameters can be found (see the Appendix for explicit formulae). These sensitivities are the only tools we have to evaluate our ability of determining the various quantities: should the I Stokes vector not vary after a perturbation of a parameter, $x$, we would be unable to infer it from the observations (it would not be a proper model parameter).

\subsection{Line sensitivities: the shape of M-E RFs}

Equations (3) and (6) provide the necessary means for studying the behavior of the M-E Stokes profiles. The shapes of the RFs do not vary dramatically from model to model or from line to line. M-E RFs appear homologous to each other. This property allows us to choose a single line to illustrate the practical usefulness of our functions. Let us take the Fe I line at $525.064 \mathrm{~nm}$ as an example. We select this line because it is used by the IMaX magnetograph (Martínez Pillet et al. 2004) and some of the results have implications either for the design or for the analysis of the data to be obtained with this magnetograph. The line has an effective Landé factor of 1.5 and is often considered to be quite insensitive to temperature perturbations (e.g., Stenflo et al. 1984). A single model is also enough for our purposes. We have used the NSO Fourier Transform Spectrometer atlas as a reference spectrum and the line was fit with errors smaller than a $2 \%$. The resulting model parameters are: $S_{0}=0.02, S_{1}=1$, $\eta_{0}=7.2, a=0.3, \Delta \lambda_{\mathrm{D}}=30 \mathrm{~m} \AA$ and a macroturbulent velocity, $v_{\text {mac }}=0.37 \mathrm{~km} \mathrm{~s}^{-1}$. Unless otherwise stated, all the numerical examples that follow refer to this line and this model. Several magnetic field strengths (200, 800, 1400 and $2000 \mathrm{G})$ have been used to synthesize the Stokes profiles and their RFs, assuming a constant field inclination and azimuth of 45 degrees.

Figure 1 shows the synthesized Stokes profiles. As the magnetic field increases, the Stokes $V$ lobes increase but their peaks do not separate much because the strong field regime has not been reached for this line with these strengths. In Fig. 2, we give a graphical illustration of the analytical RFs of the four Stokes parameters to magnetic field strength perturbations. Both the Stokes profiles and the RFs present wavelength symmetry properties, as expected from a M-E model atmosphere. The RFs to the magnetic field strength preserve the Stokes profile symmetries while velocity RFs display opposite parity (see Fig. 3).

Figure 2 shows that the response of the line is wavelength dependent. Different wavelength positions have different sensitivities. Within a single Stokes profile different wavelength samples react differently to the same perturbation. Some of the samples are insensitive. For instance, in this example the Stokes $V$ zero-crossing point remains the same regardless of $B$ and, hence, the response is zero at this wavelength. All the RFs show peaks corresponding to different maxima and minima. Note that these extrema pinpoint where the Stokes profiles are more sensitive to perturbations of the physical quantity: the greater the peak, the greater the sensitivity.

Although Stokes $I, Q$ and $U$ are more sensitive to $B$ perturbations when the strength is greater, the Stokes $V$ profile sensitivity to field strength perturbations is a maximum for the weak fields and decreases with increasing field strength. In the weak field regime, Stokes $V$ is proportional to $B$ and any change of $B$ is translated directly into an increase (or a decrease) of the $V$ signal; when the field increases, however, a competition between increasing the profile and peak separation becomes important; At a given $B$ value, peaks will no longer increase but separate from each other. This behavior of Stokes profiles is known for long but a glance to the Stokes $V$ panel of Fig. 2 illustrates it in a very clear way. Moreover, the sensitivity of Stokes $V$ in the weak field regime explains the reasonably accurate inversion results for weak magnetic fields obtained in numerical experiments by Westendorp Plaza et al. (1998).

Figure 3 shows the Stokes RFs to LOS velocity. The first clear feature in this figure is that neither the sizes nor the shapes depend on the LOS velocity. The latter only shifts the RFs as it does with the profiles. The RF size is larger for Stokes $I$ and $V$ than for Stokes $Q$ and $U$, because of the corresponding size of the profiles. Since Stokes $I$ and $V$ are larger than Stokes $Q$ and $U$ in this example, most information on velocities is carried by $I$ and $V$. The LOS velocity can always be well determined because the loss of sensitivity to $v_{\mathrm{LOS}}$ perturbations of the Stokes $I$ profile is compensated by that of the $V$ profile.

The Stokes $I$ RF to LOS velocity perturbations decreases with $B$ when the Stokes $Q, U$, and $V$ RFs increase. This is due to the different shape ratios of the various profiles. According to Cabrera Solana et al. (2005), the spectral line sensitivity to the LOS velocity is mostly determined by the ratio between the 
D. Orozco Suárez and J. C. del Toro Iniesta: The usefulness of analytic response functions
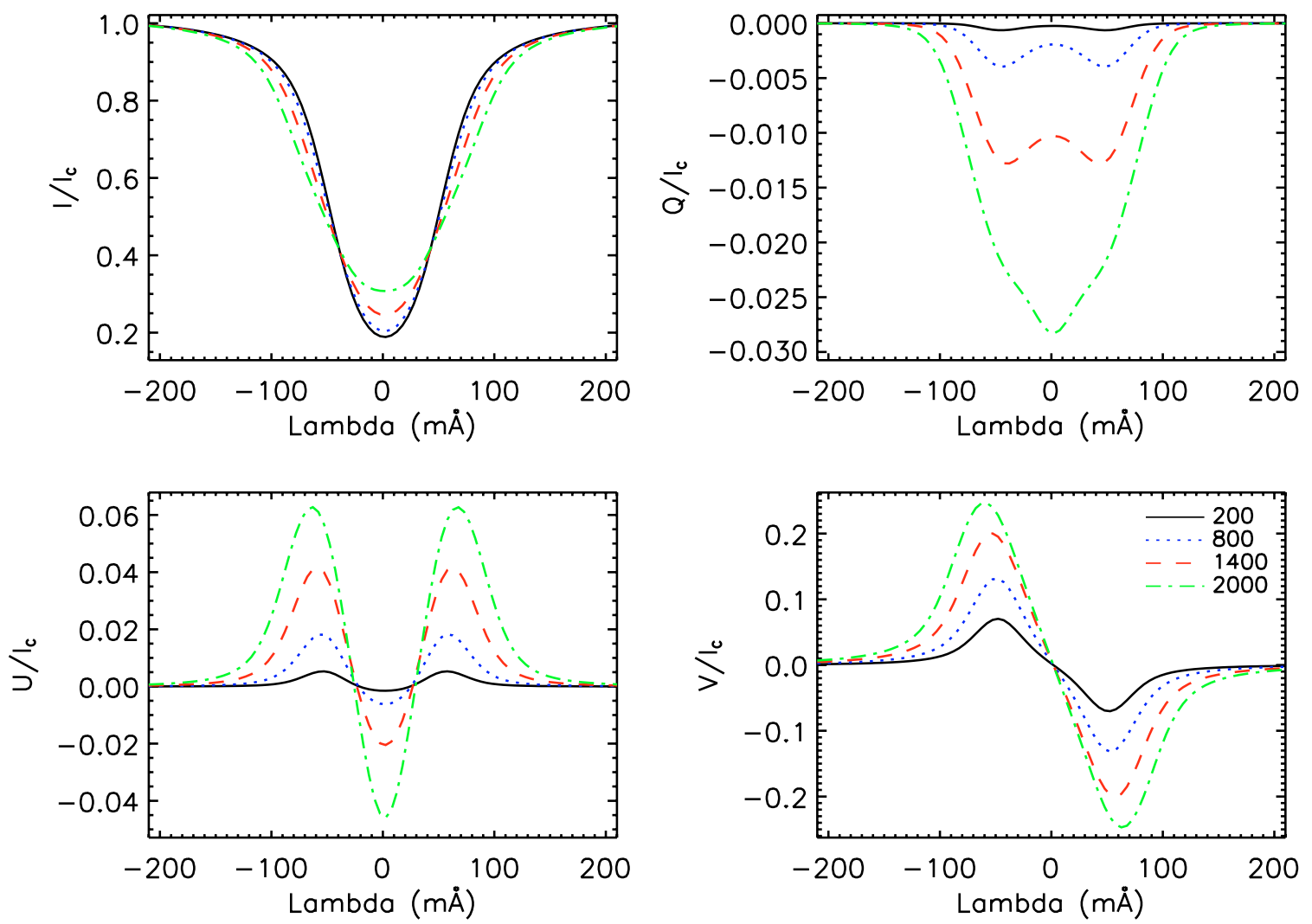

Fig. 1. Stokes $I / I_{\mathrm{c}}, Q / I_{\mathrm{c}}, U / I_{\mathrm{c}}$ and $V / I_{\mathrm{c}}$, for the Fe I line at $525.06 \mathrm{~nm}$, with a magnetic inclination and azimuth of 45 degrees. Different lines stand for different magnetic field strength values. The Stokes parameters are normalized to the local continuum.
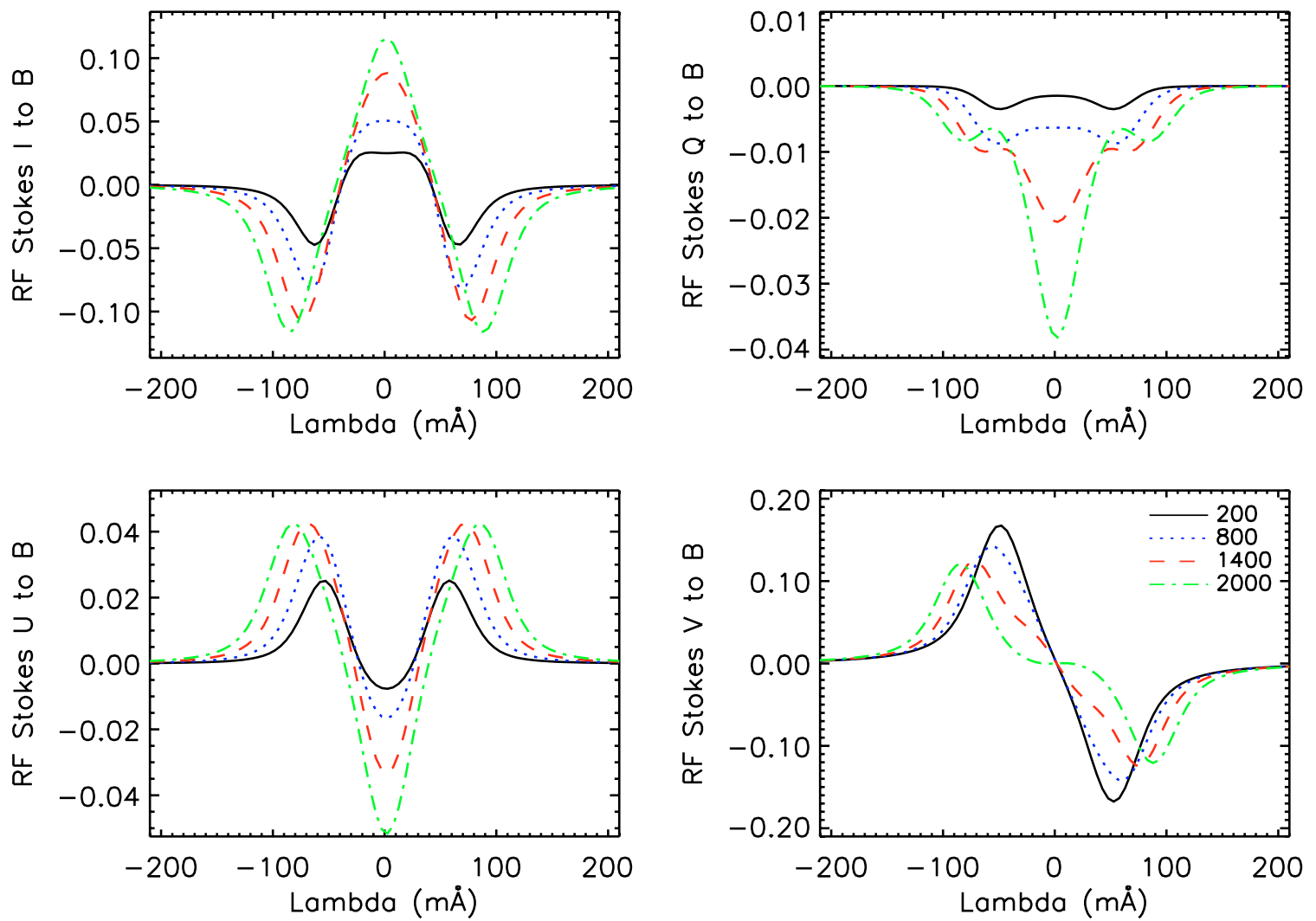

Fig. 2. Analytical M-E RFs of Stokes $I / I_{\mathrm{c}}, Q / I_{\mathrm{c}}, U / I_{\mathrm{c}}$ and $V / I_{\mathrm{c}}$ to magnetic field strength for the Fe $\mathrm{I}$ line at $525.06 \mathrm{~nm}$, with a magnetic inclination and azimuth of 45 degrees. Different lines stand for different magnetic field strength values. Units are $10^{-3} \mathrm{G}^{-1}$. 

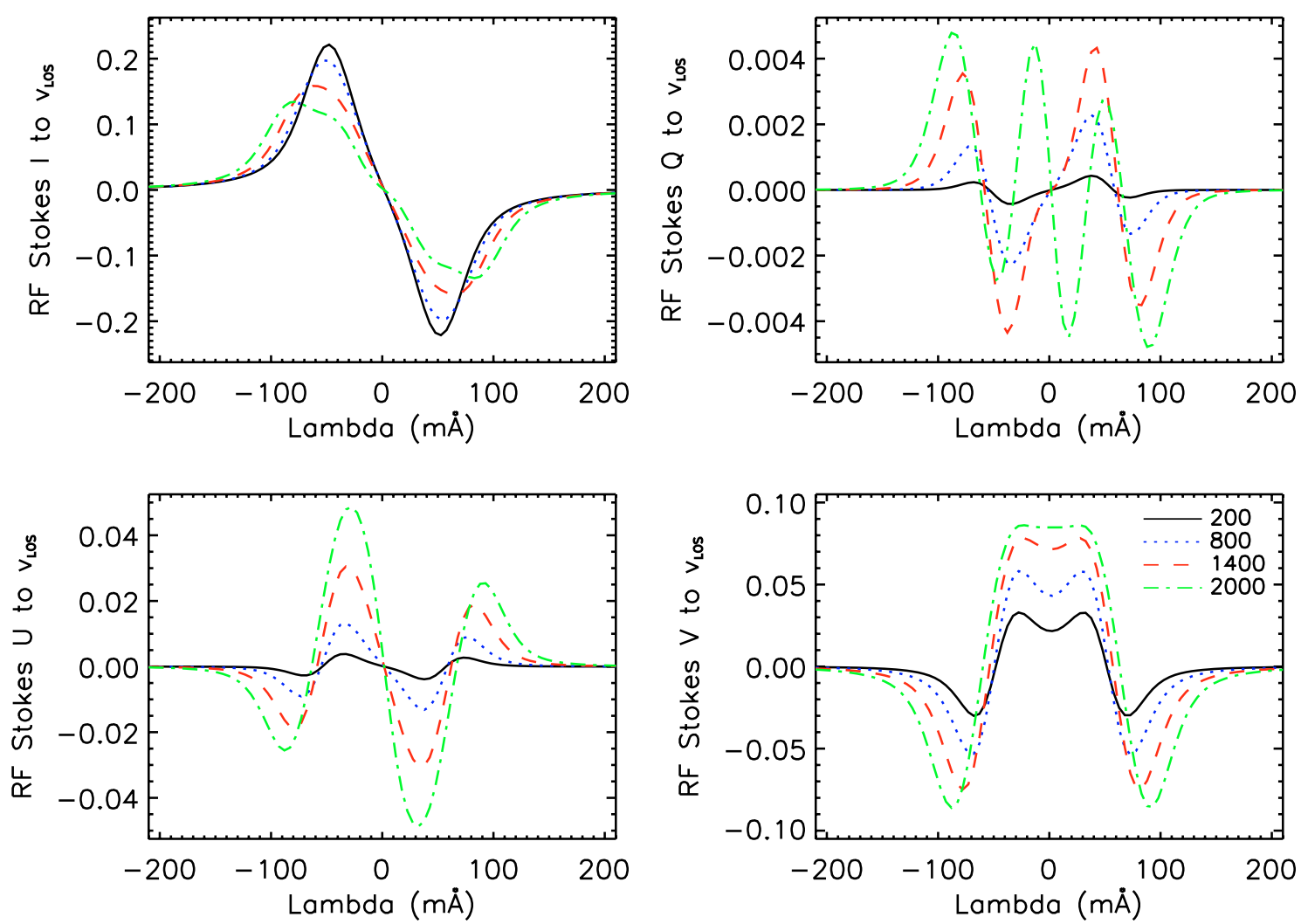

Fig. 3. Analytical M-E RFs of Stokes $I / I_{\mathrm{c}}, Q / I_{\mathrm{c}}, U / I_{\mathrm{c}}$ and $V / I_{\mathrm{c}}$ to LOS velocity for the Fe $\mathrm{I}$ line at $525.06 \mathrm{~nm}$, with a magnetic inclination and azimuth of 45 degrees. Different lines stand for different magnetic field strength values. Units are $\left[\mathrm{km} \mathrm{s}^{-1}\right]^{-1}$.

width and the depth of the line. The greater the field strength, the wider and shallower the Stokes $I$ profile. Therefore, its sensitivity to $v_{\mathrm{LOS}}$ perturbations decreases with increasing $B$. Each lobe of Stokes $V$, however, first becomes larger and then narrower and steeper at the central wavelength as $B$ increases. Hence its greater sensitivity to $v_{\mathrm{LOS}}$ for increasing field strengths.

The relative maxima of the RFs to LOS velocity perturbations correspond to wavelength positions where the inflection points of the Stokes profiles are located independently of the model atmosphere and spectral line. For instance, the minimum of Stokes $I$ and the peaks of Stokes $V$ correspond to zeros on the corresponding RFs to LOS velocity, therefore regions where the Stokes profiles do not change when LOS velocity does.

The extrema of the RFs to $B$ and to $v_{\text {LOS }}$ perturbations do not coincide with those of the corresponding profiles. This fact can be clearly seen in, e.g., the bottom right panels of Figs. 2 and 3. Therefore, the extrema of the Stokes profiles do not carry, in principle, more information on given parameters than any other particular wavelength sample. Another very interesting feature is that, for a given spectral line, the RFs differ from each other. RFs to magnetic field strength perturbations do not resemble those to LOS velocity perturbations (compare Figs. 2 and 3). For instance, their maximum sensitivities (RF peaks) are placed at different wavelengths. These differences among RFs help disentangle the influences on spectral line formation of the various model quantities and allow inversion algorithms based on RFs (e.g., SIR by Ruiz Cobo \& del Toro Iniesta 1992) to obtain accurate results: if a given Stokes profile is inappropriate for a particular wavelength sample, other profile or wavelength samples provide the required information. RF differences can also be seen for the other M-E parameters except for $\Delta \lambda_{\mathrm{D}}, \eta_{0}$ and $a$. The RFs to these thermodynamic parameter perturbations are very similar to each other as can be seen in Fig. 4. A small perturbation of any of these three parameters produces a modification in the Stokes profiles that is very similar to the changes produced by small perturbations of the other two. These similarities between the $\Delta \lambda_{\mathrm{D}}, \eta_{0}$ and $a$ RFs explain the trade-offs often observed in M-E inversions. Fortunately, their RFs are different enough from those of the other model parameters for them to be accurately retrieved (see, e.g., Westendorp Plaza et al. 1998). Thus, the M-E model atmosphere, although providing a simplistic scenario for line formation which may not full account for all thermodynamic properties, allows fairly accurate inferences of the constant magnetic field vector $\boldsymbol{B}, v_{\mathrm{LOS}}, S_{0}$ and $S_{1}$.

The RFs to magnetic field inclination and azimuth perturbations do not depend on the derivatives of the absorption and dispersion profiles; thus, the shapes of the RFs are very similar to the corresponding Stokes profiles (see Fig. 5). Only Stokes $Q$ and $U$ respond to azimuth perturbations. The larger the field strength, the greater the sensitivity of the Stokes profiles to $\gamma$ and $\chi$ perturbations. This is again an explanation of a well known fact: we measure $\gamma$ and $\chi$ more accurately when $B$ is strong.

\subsection{Relative response functions}

So far we have only discussed "absolute" RFs, i.e., functions with dimensions; e.g. the RF to $B$ is measured in $\mathrm{G}^{-1}$, that to $v_{\text {LOS }}$ perturbation is measured in $\left(\mathrm{km} \mathrm{s}^{-1}\right)^{-1}$ and so on: RFs give modifications of the profile per unit perturbation of the parameter. To compare them to one another, relative RFs should be used (Ruiz Cobo \& del Toro Iniesta 1994; del Toro Iniesta \& Ruiz Cobo 1996). These relative responses are obtained by multiplying the standard, absolute RFs by the corresponding model parameter. Relative RFs tell us how sensitive one model 
D. Orozco Suárez and J. C. del Toro Iniesta: The usefulness of analytic response functions
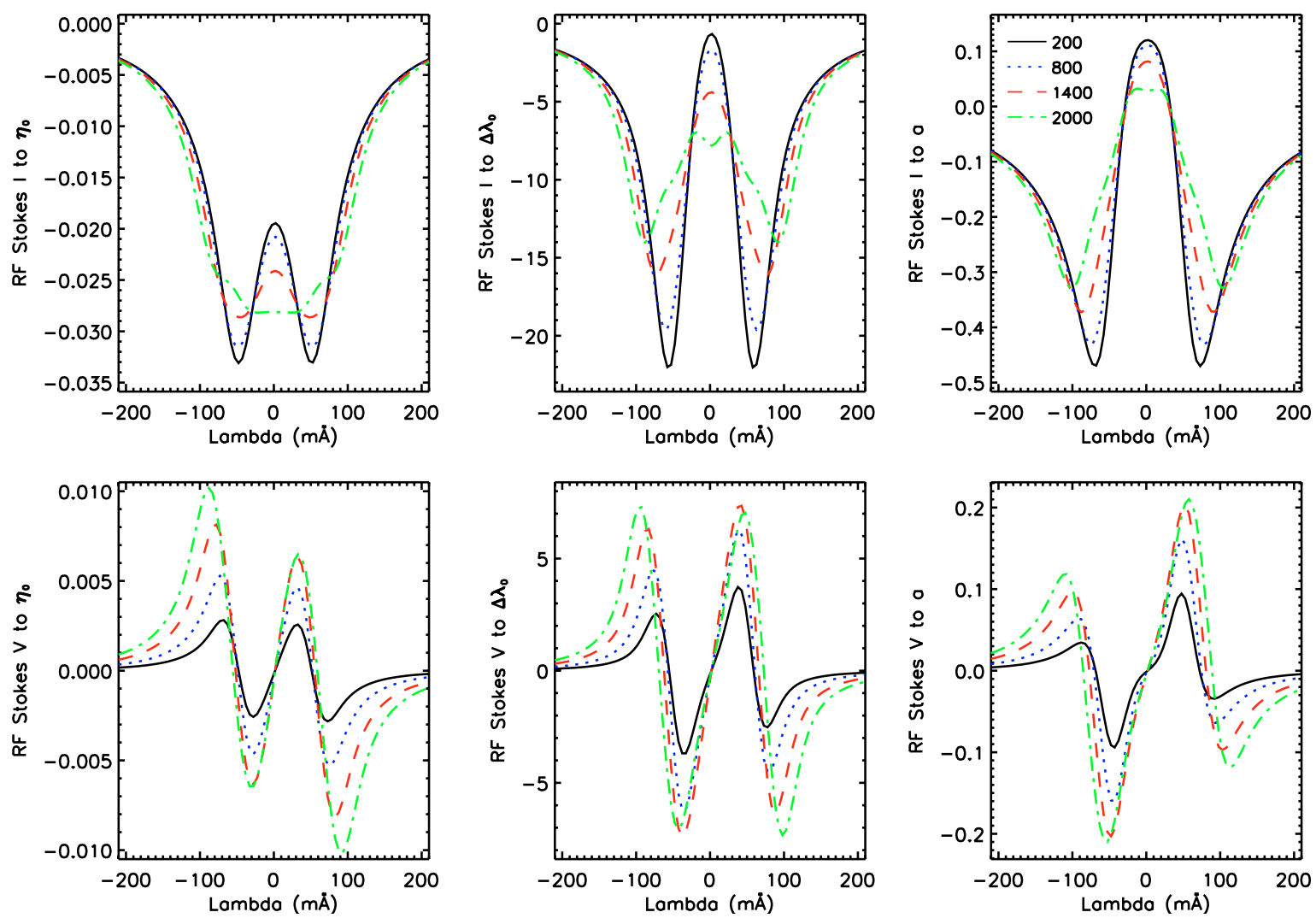

Fig. 4. Analytical M-E RFs of Stokes $I / I_{\mathrm{c}}$ (upper panels) and $V / I_{\mathrm{c}}$ (bottom panels) to $\eta_{0}$, to $\Delta \lambda_{\mathrm{D}}$ and to $a$ (left, middle and right panels respectively), for the Fe I line at $525.06 \mathrm{~nm}$, with a magnetic inclination and azimuth of 45 degrees. Different lines stand for different magnetic field strength values. Units are dimensionless for the left and right panels since $\eta_{0}$ and $a$ are dimensionless. Units for the middle panels are $\AA^{-1}$. Note the similarities among the different RFs.
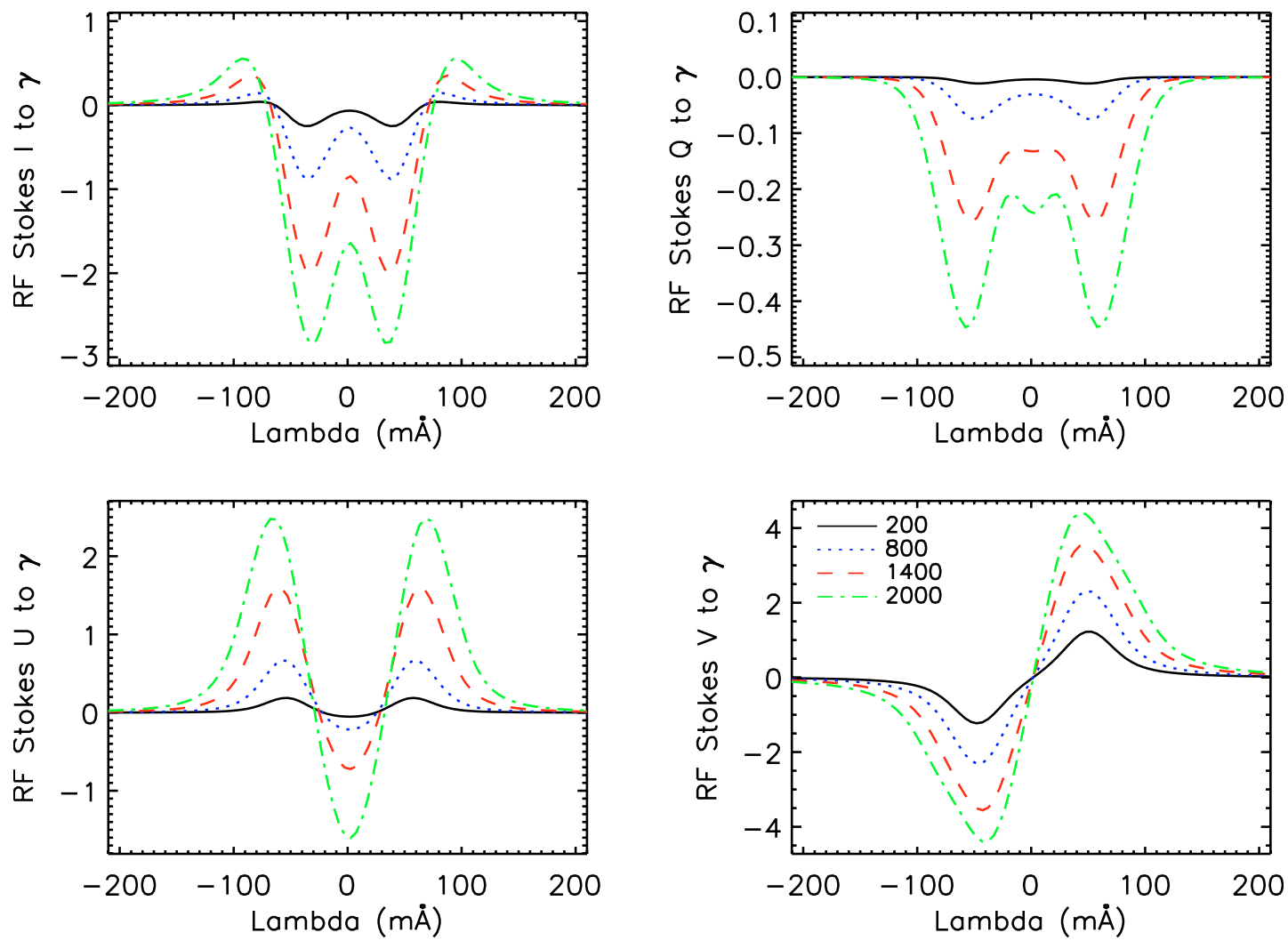

Fig. 5. Analytical M-E RFs of Stokes $I / I_{\mathrm{c}}, Q / I_{\mathrm{c}}, U / I_{\mathrm{c}}$ and $V / I_{\mathrm{c}}$ to magnetic field inclination, $\gamma$, for the Fe I line at $525.06 \mathrm{~nm}$, with a magnetic inclination and azimuth of 45 degrees. Different lines stand for different magnetic field strength values. Units are in $10^{-3}[\mathrm{degrees}]^{-1}$. 

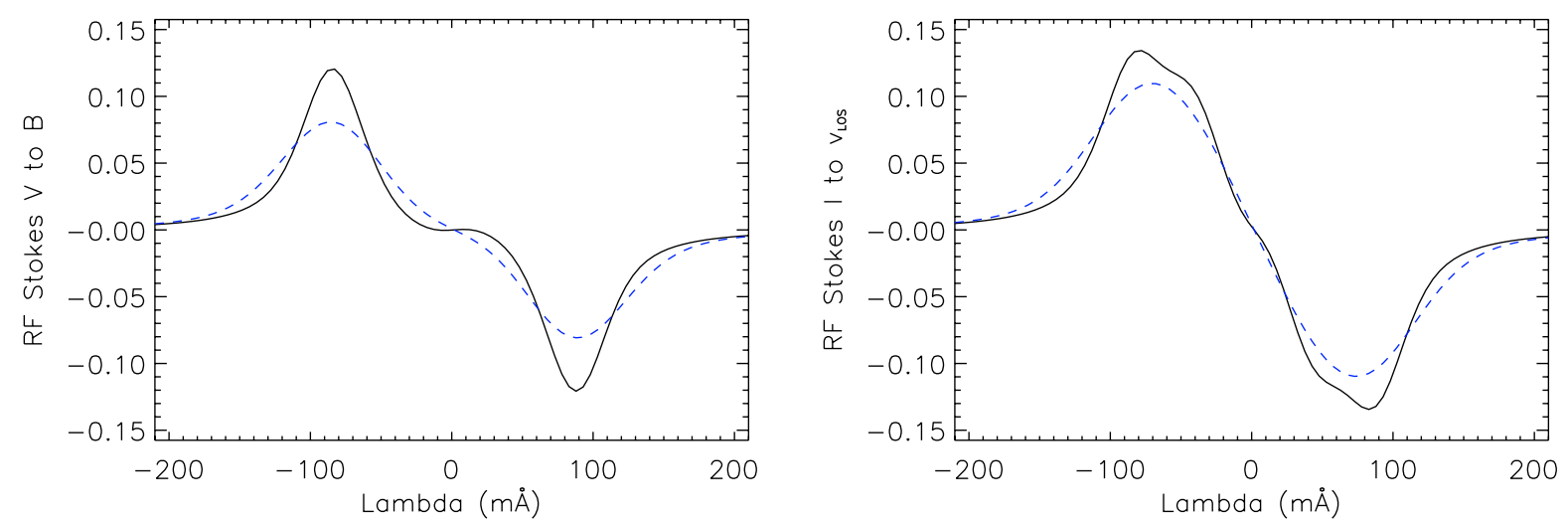

Fig. 6. Analytical M-E RFs of Stokes $V / I_{\mathrm{c}}$ to the magnetic field strength (left panel) and of Stokes $I / I_{\mathrm{c}}$ to the LOS velocity (right panel) for the $\mathrm{Fe}$ I line at $525.06 \mathrm{~nm}$, with a magnetic field strength of $2000 \mathrm{G}$ and field inclination and azimuth of 45 degrees. The dashed lines correspond to the RFs convolved with a Gaussian smearing profile of $60 \mathrm{~m} \AA$ of full width at half maximum (FWHM). Solid lines correspond to the original RFs. Units are in $10^{-3} \mathrm{G}^{-1}($ left $)$ and $\left[\mathrm{km} \mathrm{s}^{-1}\right]^{-1}$ (right).

parameter is compared to the others. For instance, the relative RF to $\Delta \lambda_{\mathrm{D}}$ is much larger than that to $\eta_{0}$ and that to $a$ (in particular three times as large as the RFs to $\eta_{0}$ and twenty times larger than those to $a$ for Stokes $I$, in our sample M-E atmosphere). This means that a small relative perturbation of $\Delta \lambda_{D}$ changes the Stokes profiles much more than the same relative perturbation of $\eta_{0}$ or $a$. Consequently, $\Delta \lambda_{\mathrm{D}}$ should be better determined by M-E inversion codes.

\subsection{Two-component model atmospheres}

Model atmospheres with two or more components are commonly used in the analysis of observations. Any two-component model atmosphere is based on the assumption that within the resolution element two different atmospheres coexist, namely, one magnetic atmosphere filling a surface fraction $\alpha$, and one nonmagnetic atmosphere filling the remaining $(1-\alpha)$ fraction. $\alpha$ is called the magnetic filling factor. If $\boldsymbol{I}_{\mathrm{m}}$ stands for the Stokes profile vector emerging from the magnetic region and $\boldsymbol{I}_{\mathrm{nm}}$ for that of the non-magnetized atmosphere, the observed Stokes vector can be written as $\boldsymbol{I}=\boldsymbol{I}_{\mathrm{m}} \alpha+\boldsymbol{I}_{\mathrm{nm}}(1-\alpha)$.

Thus, according to Eq. (6), the RFs to $\alpha$ perturbations are given by $\boldsymbol{I}_{\mathrm{m}}-\boldsymbol{I}_{\mathrm{nm}}$. Hence, the larger the difference between the magnetic and the non-magnetic atmospheres, the greater the sensitivity to $\alpha$. Since most of the difference is the polarization signal itself, $Q_{\mathrm{m}}, U_{\mathrm{m}}, V_{\mathrm{m}}$, when this signal is strong we can easily discern it from the non-magnetic signal.

\subsection{The influence of smearing}

Spectral line smearing by macroturbulence is a well known effect that needs be taken into account in the analysis of most observations except, perhaps, in those with very high spatial resolution (Asplund et al. 2000). Besides macroturbulence, instruments have finite-width profiles that produce smearing of the observed Stokes profiles which become wider and with smaller peaks. This smearing reduces the information on physical parameters carried by the spectral line through convolution: $\boldsymbol{I}_{\mathrm{obs}}=$ $\boldsymbol{I} * F(\lambda)$, where $*$ stands for the convolution symbol and the scalar smearing profile, $F(\lambda)$, is convolved with all the four Stokes parameters.

This loss of information through smearing is also translated into a loss of sensitivity of spectral lines to the atmospheric quantities. In fact, since the derivative of a convolution is equal to the convolution of the derivative of one of the functions with the second one, response functions become smeared as well:

$\boldsymbol{R}_{\mathrm{obs}, x}=\boldsymbol{R}_{x} * F(\lambda)$

Figure 6 shows the effect of RF smearing. The convolved RFs are smoother and significant information is lost.

\subsection{The influence of noise}

Stokes profiles are affected by the noise intrinsic to the observational process. Should the polarimetric signal be buried by noise, any algorithm one could devise to determine atmospheric quantities would fail. Therefore, our ability to infer accurate solar parameters depend significantly on the signal-to-noise ratio of the observations.

Response functions can help in quantifying this effect. Since RFs simply provide the modification of the Stokes profiles after a perturbation of the physical quantities, if that modification is smaller than the noise level it will be effectively undetectable. In other words, the size of RFs to perturbations of a given quantity sets a threshold for the detection of a unit of such a quantity: for instance, according to Fig. 2, 1 Gauss will only be detectable by a single wavelength sample if the noise is below $1.5 \times 10^{-4}$ (continuum intensity is at 1); within the linear approximation ${ }^{1}$, 10 Gauss will be detectable with a noise below $1.5 \times 10^{-3}$ and so on.

According to Eq. (6), a standard deviation in the Stokes signal $\sigma$ will induce an error $\sigma_{x}$ per single wavelength sample given by:

$\sigma=\sqrt{R_{1, x}^{2}+R_{2, x}^{2}+R_{3, x}^{2}+R_{4, x}^{2}} \sigma_{x}$.

Detectability should increase, of course, as the root of the wavelength sample number, but the RFs allow an estimate of the expected accuracy of our inferences.

The above estimates can be considered lower limits for the errors since model parameters are not independent of each other and correlations may exist between sensitivities such as those already reported between $\eta_{0}, \Delta \lambda_{\mathrm{D}}$ and $a$.

${ }^{1}$ RFs come from a linear perturbation analysis of the radiative transfer equation. 

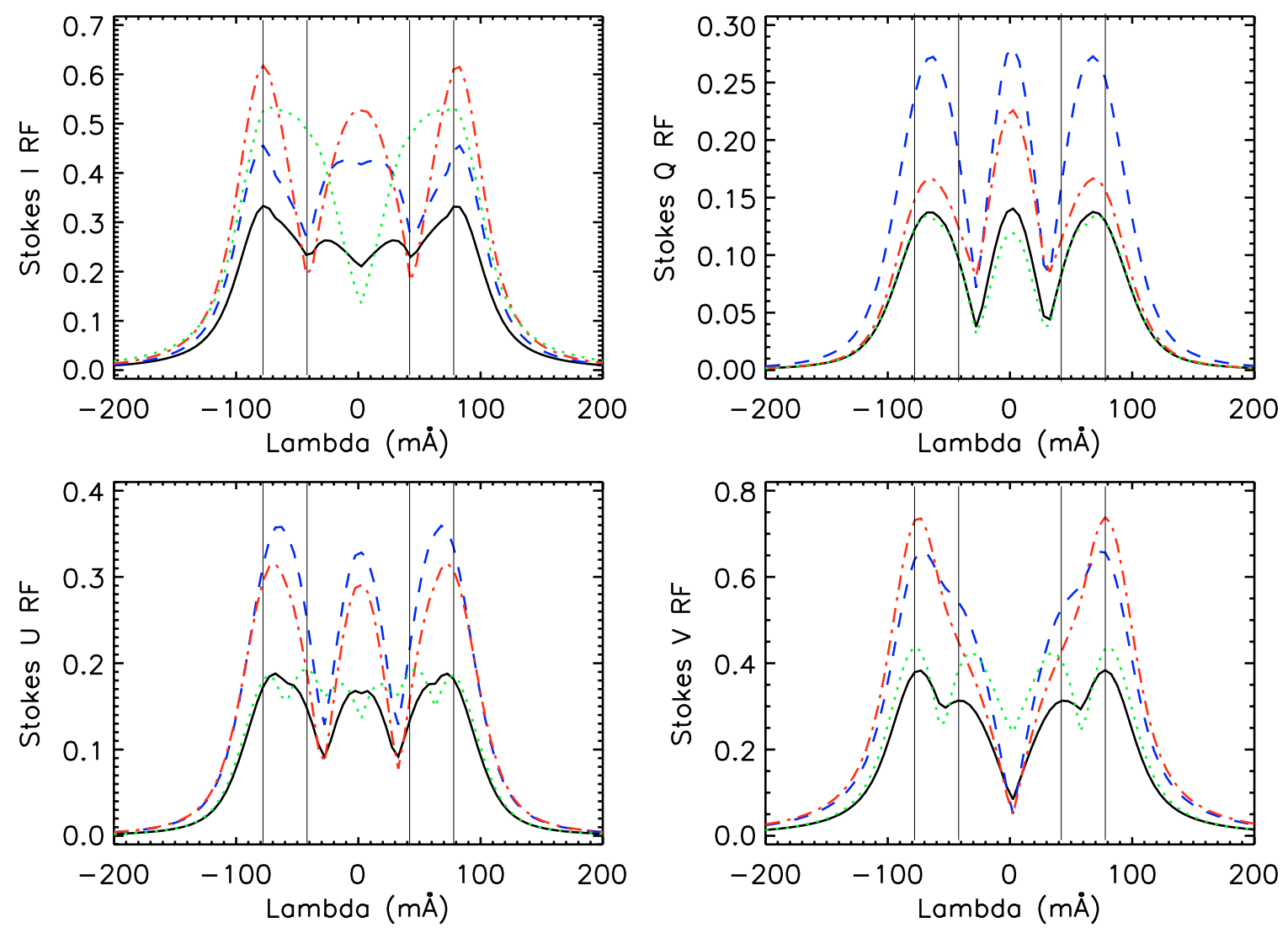

Fig. 7. Four different linear combinations of the Stokes vector RFs for the IMaX line. The plotted curves correspond to $\alpha_{1,2,3,4}=1,1,1,1$ (solid, black lines), $\alpha_{1,2,3,4}=2,2,2,0.5$ (dashed, blue lines), $\alpha_{1,2,3,4}=3,1,1,0.5$ (dashed-dotted, red lines), and $\alpha_{1,2,3,4}=0.5,1,1,3$ (dotted, green lines). The light-grey, vertical lines indicate a possible choice for wavelength sampling $( \pm 42,78 \mathrm{~m} \AA)$.

\section{The usefulness of the RFs for vector magnetographs}

Modern vector magnetographs are not restricted to using one or two wavelength samples as are the classical magnetographs. Instruments like IMaX are devised to measure up to five wavelengths: one in the continuum and four across the line profile. The choices of the spectral line, of the number of samples and of the precise wavelength for each of them are important issues that arise during the design phase of the instrument. This section is aimed at illustrating how the RFs can help such decisions.

Finding a suitable spectral line is crucial and can be achieved through RFs on the simple phenomenological model by Cabrera Solana et al. (2005) that allows establishing a ranking of sensitivities to the different atmospheric parameters among the various lines considered. The IMaX Fe I line at $525.064 \mathrm{~nm}$ can be seen in Fig. 8. Data for this line have been included in the original figure by Cabrera Solana et al. (2005), where it is identified as one of the most sensitive of the set to velocity perturbations. It has a medium sensitivity to magnetic field strength perturbations in both the strong and the weak field regimes. However, it is not very sensitive to temperature (not shown) and, hence, a good candidate for inferences in the various solar structures avoiding thermodynamic trade-offs. The Helioseismic and Magnetic Imager (HMI; Scherrer \& SDO/HMI Team 2002) and the Visible-light Imager and Magnetograph (VIM; Marsch et al. 2005), two planned instruments for the Solar Dynamics Observatory, NASA, and the Solar Orbiter, ESA, missions, will use the Fe I line at $617.334 \mathrm{~nm}$. This spectral line is very well ranked in Fig. 8 for inference of both magnetic field strengths and $\mathrm{LOS}$ velocities.

A minimum number of wavelength samples is obtained by roughly doubling the free parameters of the model: since an M-E model is made up of just ten parameters, a minimum of twenty observables (five wavelengths times the four Stokes parameters) is needed. This is the choice for all the three instruments mentioned above. Unfortunately, no purely objective means exists to select the wavelengths for the samples. Nevertheless, RFs are a powerful tool that help select those wavelengths that better suit our purposes. If one is interested, for instance, in just the magnetic field strength and neglects the other physical quantities, choosing those wavelengths where the RFs to $B$ reach local maxima would be appropriate. If the interest lies in several physical quantities at the same time (e.g. on the three components of the magnetic field and on the LOS velocity) we suggest the use of a linear combination of regular RFs weighted according to the specific interests. Since RFs can be positive or negative, we propose the use of absolute-valued RFs. Hence, consider

$\mathcal{R}_{j}=\sum_{i} \alpha_{i}\left|R_{j, i}\right|$

where $j$ runs from 1 through 4 , corresponding to the four Stokes parameters, and index $i$ accounts for the physical parameters. Since the set of weights $\alpha_{i}$ can be tailored at will, there is no single choice for samples but an examination of $\mathcal{R}$ provides important hints for the selection. As an example, Fig. 7 shows different linear combinations for the IMaX line case. If index $i$ runs from 1 through 4 standing for $B, \gamma, \phi$, and $v_{\mathrm{LOS}}$, respectively, the plotted 

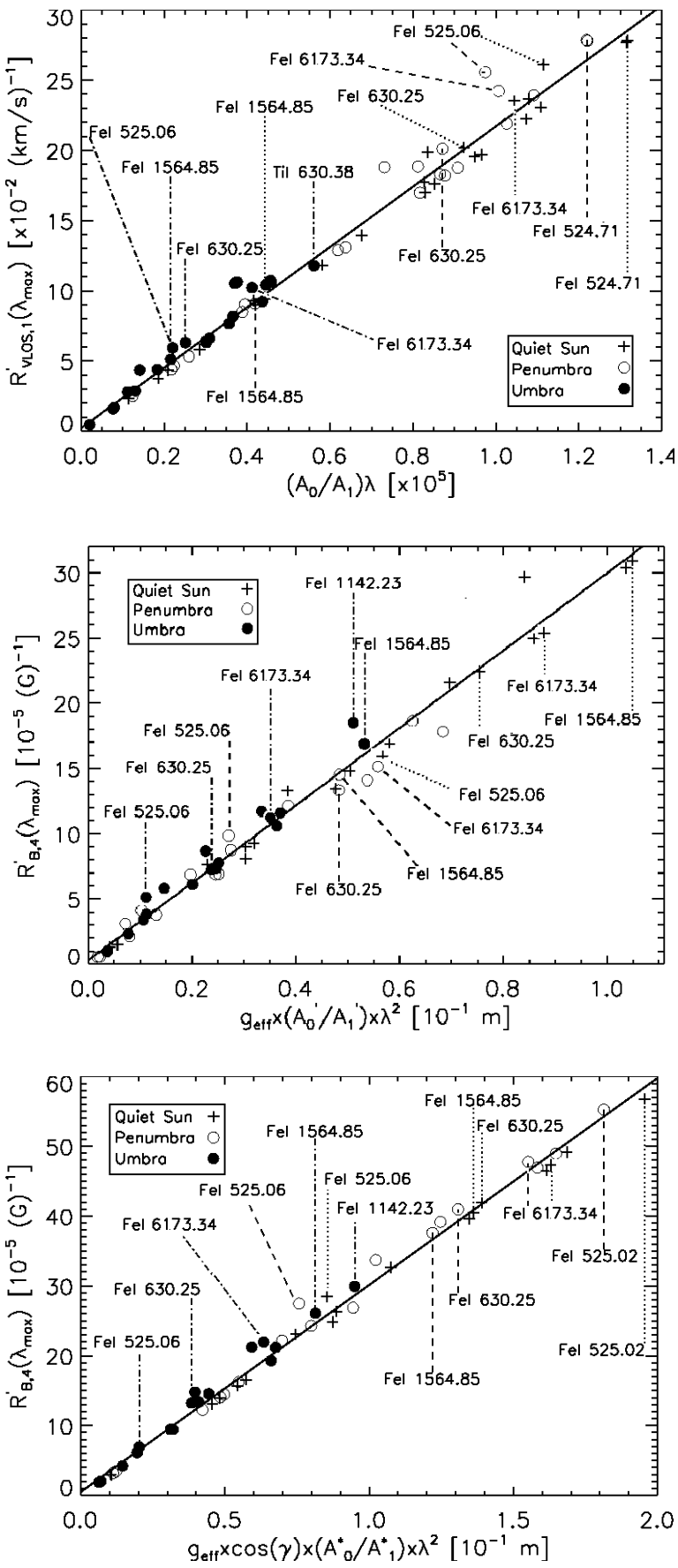

Fig. 8. Upper panel: maximum value of the $\tau$-integrated RF to $v_{\mathrm{LOS}}$ for different lines as a function of the shape ratio multiplied by the central wavelength of the transition (see Cabrera Solana et al. 2005). Middle panel: maximum values of the integrated RF to $B$ for the same set of lines with $g_{\mathrm{eff}} \neq 0$, as a function of the shape ratio multiplied by the squared central wavelength (strong field regime). Bottom panel: maximum values of the integrated RF to $B$ (weak field regime). The sensitivities have been evaluated in the quiet Sun (crosses), penumbral (circles) and hot umbral (filled circles) model atmospheres. Dotted, dashed, and dash-dotted lines mark specific transitions in the quiet sun, penumbral, and umbral models, respectively.

curves correspond to $\alpha_{1,2,3,4}=1,1,1,1$ (solid, black lines), $\alpha_{1,2,3,4}=2,2,2,0.5$ (dashed, blue lines), $\alpha_{1,2,3,4}=3,1,1,0.5$ (dashed-dotted, red lines), and $\alpha_{1,2,3,4}=0.5,1,1,3$ (dotted, green lines). The vertical lines indicate a possible choice for wavelength sampling $( \pm 42,78 \mathrm{m \AA})$, selected mostly from the properties of the Stokes $I$ and $V$ RFs since these two parameters usually exhibit the largest signals in solar atmospheres. While the most external samples seem to be optimum, some other good choices for the inner wavelengths are possible and up to the user.

\section{Conclusions}

The many interesting features of analytic response functions have been discussed in this paper by considering the specific case of an M-E model atmosphere. Since an analytic solution for the radiative transfer equation is available for this atmosphere, the sensitivities of spectral lines, as described by RFs, can also be cast in an analytical form by simply taking partial derivatives of such a solution with respect to the model parameters. The analytic M-E solution has been thoroughly used in the past for insight into radiative transfer physics and as a purely practical diagnostic tool through the M-E inversion codes. Likewise, we have shown in this paper that the analytic, M-E RFs are useful to better understand spectral line formation and the behavior of Stokes profiles in different formation conditions and also for practical recipes that can help in selecting spectral lines for given purposes, in selecting wavelength samples, etc.

A summary of the various results obtained follows:

1. Response functions look homologous to each other, hence enabling qualitative, general discussions by considering a single spectral line in a specific model atmosphere. Here, we have targeted the IMaX, Fe I line at $525.60 \mathrm{~nm}$ in a M-E model representative of the quiet Sun thermodynamics (as observed by FTS) and with various vector magnetic fields and LOS velocities.

2. The sensitivities of spectral lines to the various parameters depend on wavelength: some samples are better suited to diagnose a given parameter; some wavelengths are even insensitive to another parameter. The RF extrema show trivially those wavelengths where sensitivity is maximum.

3. As expected in M-E conditions where no gradient of LOS velocity is present, RFs display clear wavelength symmetry properties. The RFs to magnetic field strength perturbations show similar parity as the Stokes profiles while the RFs to LOS velocity perturbations are of opposite parity.

4. Stokes $V$ sensitivities to $B$ perturbations are significant for very weak field strengths. This fact explains the reasonably accurate results of M-E inversions in this strength regime.

5. The shape of the RFs to LOS velocity perturbations does not depend on $v_{\text {LOS }}$ except for the Doppler shift. Variations of sensitivity of the Stokes $I$ and $V$ profiles are compensated: when information on $v_{\text {LOS }}$ decreases in Stokes $I$ it increases in Stokes $V$, so that $v_{\text {LOS }}$ remains well inferred in any circumstance.

6. We understand the trade-offs often found in the inversion codes among M-E thermodynamic parameters: their corresponding RFs are very similar to each other. Fortunately, they are different from the other RFs and can accurately infer vector magnetic fields and LOS velocities. Among the thermodynamic parameters, the relative sensitivity to $\Delta \lambda_{D}$ perturbations is larger than that to $\eta_{0}$ and $a$, hence enabling better inferences.

7. The magnetic filling factor $\alpha$ is better determined if there are significant differences between magnetic and non-magnetic atmospheres. When $B$ is large this result is natural; when $B$ is small, this result explains that differences in the thermodynamics of both atmospheres can help in inferring $\alpha$ properly. 
8. Direct estimates of affordable noise levels can be directly obtained from RFs.

9. Response functions can also be used to select spectral lines for given purposes or for given measurements. Moreover, a suitable combination of RFs provides quantitive arguments for wavelength sample choice.

Acknowledgements. Interesting discussions with D. Cabrera Solana and L. R Bellot Rubio are thanked. This work has been partially funded by Spanish Ministerio de Educación y Ciencia through Project No. ESP2003-07735-C0403 including a percentage from European FEDER funds.

\section{References}

Asplund, M., Nordlund, Å., Trampedach, R., Allende Prieto, C., \& Stein, R. F. 2000, A\&A, 359, 729

Cabrera Solana, D., Bellot Rubio, L. R., \& del Toro Iniesta, J. C. 2005, A\&A, 439,687

Landi degl'Innocenti, E. 1976, A\&AS, 25, 379
Landi Deglinnocenti, E., \& Landi Deglinnocenti, M. 1977, A\&A, 56, 111

Landolfi, M., \& Degl'Innocenti, E. L. 1982, Sol. Phys., 78, 355

Marsch, E., Marsden, R., Harrison, R., Wimmer-Schweingruber, R., \& Fleck, B. 2005, Adv. Space Res., 36, 1360

Martínez Pillet, V., Collados, M., Sánchez Almeida, J., et al. 1999, High Resolution Solar Physics: Theory, Observations, and Techniques, ASP Conf. Ser., 183, 264

Martínez Pillet, V., Bonet, Jose A., Collados, M. V., et al. 2004, Proc. SPIE, 5487, 1152

Mein, P. 1971, Sol. Phys., 20, 3

Rachkovsky, D. N. 1962, Izv. Krymskoi Astrofiz. Obs., 27, 148

Rachkovsky, D. N. 1967, Izv. Krymskoi Astrofiz. Obs., 37, 56

Ruiz Cobo, B., \& del Toro Iniesta, J. C. 1992, ApJ, 398, 375

Ruiz Cobo, B., \& del Toro Iniesta, J. C. 1994, A\&A, 283, 129

Scherrer, P. H., \& SDO/HMI Team 2002, BAAS, 34, 735

Stenflo, J. O., Solanki, S., Harvey, J. W., \& Brault, J. W. 1984, A\&A, 131, 333

del Toro Iniesta, J. C. 2003, Introduction to Spectropolarimetry (Cambridge, UK:

Cambridge University Press)

del Toro Iniesta, J. C., \& Ruiz Cobo, B. 1996, Sol. Phys., 164, 169

Unno, W. 1956, PASJ, 8, 108

Westendorp Plaza, C., del Toro Iniesta, J. C., Ruiz Cobo, B., et al. 1998, ApJ, 494, 453 
D. Orozco Suárez and J. C. del Toro Iniesta: The usefulness of analytic response functions, Online Material $p 1$

\section{Online Material}


D. Orozco Suárez and J. C. del Toro Iniesta: The usefulness of analytic response functions, Online Material p 2

\section{Appendix A: Explicit formulae}

The propagation matrix $\mathbf{K}$ of the RTE can be cast in the form (e.g. del Toro Iniesta 2003)

$\mathbf{K}=\left(\begin{array}{cccc}\eta_{I} & \eta_{Q} & \eta_{U} & \eta_{V} \\ \eta_{Q} & \eta_{I} & \rho_{V} & -\rho_{U} \\ \eta_{U} & -\rho_{V} & \eta_{I} & \rho_{Q} \\ \eta_{V} & \rho_{U} & -\rho_{Q} & \eta_{I}\end{array}\right)$,

where

$\eta_{I}=1+\frac{\eta_{0}}{2}\left[\phi_{p} \sin ^{2} \gamma+\frac{\phi_{b}+\phi_{r}}{2}\left(1+\cos ^{2} \gamma\right)\right]$,

$\eta_{Q}=\frac{\eta_{0}}{2}\left[\phi_{p}-\frac{\phi_{b}+\phi_{r}}{2}\right] \sin ^{2} \gamma \cos 2 \chi$

$\eta_{U}=\frac{\eta_{0}}{2}\left[\phi_{p}-\frac{\phi_{b}+\phi_{r}}{2}\right] \sin ^{2} \gamma \sin 2 \chi$,

$\eta_{V}=\frac{\eta_{0}}{2}\left[\phi_{r}-\phi_{b}\right] \cos \gamma$

$\rho_{Q}=\frac{\eta_{0}}{2}\left[\psi_{p}-\frac{\psi_{b}+\psi_{r}}{2}\right] \sin ^{2} \gamma \cos 2 \chi$,

$\rho_{U}=\frac{\eta_{0}}{2}\left[\psi_{p}-\frac{\psi_{b}+\psi_{r}}{2}\right] \sin ^{2} \gamma \sin 2 \chi$,

$\rho_{V}=\frac{\eta_{0}}{2}\left[\psi_{r}-\psi_{b}\right] \cos \gamma$,

and $\phi_{p, b, r}$ and $\psi_{p, b, r}$ are the absorption and dispersion profiles, the $p, b, r$ indices stand for the $\pi$ and $\sigma$ components of a Zeeman multiplet, and $\eta_{0}$ is the ratio of line to continuum absorption coefficients.

$\phi_{p, b, r}$ and $\psi_{p, b, r}$ can be written as a sum of as many absorption and dispersion profiles as the number of $p, b, r$ components as follows:

$$
\begin{aligned}
\phi_{j} & =\sum_{M_{1}-M_{\mathrm{u}}=j} S_{M_{\mathrm{l}} M_{\mathrm{u}}, j} H(a, v), \\
\psi_{j} & =2 \sum_{M_{\mathrm{l}}-M_{\mathrm{u}}=j} S_{M_{\mathrm{l}} M_{\mathrm{u}}, j} F(a, v),
\end{aligned}
$$

$S_{M_{1} M_{\mathrm{u}}, j}$ being the strength of each component with $j=-1,0,1$ corresponding to $b, p$ and $r . v$ stands for the wavelength in Doppler units which follows

$v=\frac{\lambda-\lambda_{0}}{\Delta \lambda_{\mathrm{D}}}+\frac{\Delta \lambda_{\mathrm{B}}}{\Delta \lambda_{\mathrm{D}}}-\frac{\lambda_{0} v_{\mathrm{LOS}}}{c \Delta \lambda_{\mathrm{D}}}$

The wavelength shift of the different Zeeman components with respect to the original position is given by

$\Delta \lambda_{\mathrm{B}}=\frac{e \lambda_{0}^{2} B}{4 \pi m c^{2}}\left(g_{1} M_{1}-g_{\mathrm{u}} M_{\mathrm{u}}\right)$,

where 1 and $\mathrm{u}$ stand for the lower and upper levels of the line transition, $g$ for the level Landé factor, and $M$ for the magnetic level quantum number.

The evaluation of RFs reduces to the derivatives of the Stokes vector, $\boldsymbol{I}=(I, Q, U, V)$, with respect to the nine parameters, $\left(B_{0}, B_{1}, \eta_{0}, B, \gamma, \chi, \Delta \lambda_{\mathrm{D}}, V_{\mathrm{LOS}}, a\right)$. In order to easily show such derivatives suppose a generic parameter $x$. Then,

$$
\begin{aligned}
& \frac{\partial I}{\partial x}=B_{1} \mu\left(T_{1} \frac{\partial \eta_{I}}{\partial x}+\eta_{I} \frac{\partial T_{1}}{\partial x}-\Delta^{-1} \eta_{I} T_{1} \frac{\partial \Delta}{\partial x}\right) \Delta^{-1} \\
& \frac{\partial Q}{\partial x}=-B_{1} \mu\left(\frac{\partial T_{2}}{\partial x}+\frac{\partial \rho_{Q}}{\partial x} \Pi+\rho_{Q} \frac{\partial \Pi}{\partial x}-\Delta^{-1} \frac{\partial \Delta}{\partial x}\left[T_{2}+\rho_{Q} \Pi\right]\right) \Delta^{-1}
\end{aligned}
$$

$$
\begin{aligned}
& \frac{\partial U}{\partial x}=-B_{1} \mu\left(\frac{\partial T_{3}}{\partial x}+\frac{\partial \rho_{U}}{\partial x} \Pi+\rho_{U} \frac{\partial \Pi}{\partial x}-\Delta^{-1} \frac{\partial \Delta}{\partial x}\left[T_{3}+\rho_{U} \Pi\right]\right) \Delta^{-1}, \\
& \frac{\partial V}{\partial x}=-B_{1} \mu\left(\frac{\partial T_{4}}{\partial x}+\frac{\partial \rho_{V}}{\partial x} \Pi+\rho_{V} \frac{\partial \Pi}{\partial x}-\Delta^{-1} \frac{\partial \Delta}{\partial x}\left[T_{4}+\rho_{V} \Pi\right]\right) \Delta^{-1},
\end{aligned}
$$

where for simplicity

$T_{1}=\eta_{I}^{2}+\rho_{Q}^{2}+\rho_{U}^{2}+\rho_{V}^{2}$,

$T_{2}=\eta_{I}^{2} \eta_{Q}+\eta_{I}\left(\eta_{V} \rho_{U}-\eta_{U} \rho_{V}\right)$,

$T_{3}=\eta_{I}^{2} \eta_{U}+\eta_{I}\left(\eta_{Q} \rho_{V}-\eta_{V} \rho_{Q}\right)$,

$T_{4}=\eta_{I}^{2} \eta_{V}+\eta_{I}\left(\eta_{U} \rho_{Q}-\eta_{Q} \rho_{U}\right)$,

$T_{5}=\eta_{I}^{2}-\eta_{Q}^{2}-\eta_{U}^{2}-\eta_{V}^{2}+\rho_{Q}^{2}+\rho_{U}^{2}+\rho_{V}^{2}$.

$\Delta$ and $\Pi$ are defined in Eqs. (4) and (5), respectively. Their derivatives are thus given by

$$
\begin{aligned}
\frac{\partial \Delta}{\partial x}= & 2 \eta_{I} \frac{\partial \eta_{I}}{\partial x} T_{5}+\eta_{I}^{2} \frac{\partial T_{5}}{\partial x}-2 \Pi \frac{\partial \Pi}{\partial x}, \\
\frac{\partial \Pi}{\partial x}= & \eta_{Q} \frac{\partial \rho_{Q}}{\partial x}+\frac{\partial \eta_{Q}}{\partial x} \rho_{Q}+\eta_{U} \frac{\partial \rho_{U}}{\partial x}+\frac{\partial \eta_{U}}{\partial a} \rho_{U} \\
& +\eta_{V} \frac{\partial \rho_{V}}{\partial x}+\frac{\partial \eta_{V}}{\partial x} \rho_{V} .
\end{aligned}
$$

The derivatives of $T_{1}, \ldots, T_{5}$ are given by

$$
\begin{aligned}
\frac{\partial T_{1}}{\partial x}= & 2\left(\eta_{I} \frac{\partial \eta_{I}}{\partial x}+\rho_{Q} \frac{\partial \rho_{Q}}{\partial x}+\rho_{U} \frac{\partial \rho_{U}}{\partial x}+\rho_{V} \frac{\partial \rho_{V}}{\partial x}\right) \\
\frac{\partial T_{2}}{\partial x}= & 2 \eta_{I} \frac{\partial \eta_{I}}{\partial x} \eta_{Q}+\eta_{I}^{2} \frac{\partial \eta_{Q}}{\partial x}+\frac{\partial \eta_{I}}{\partial x}\left(\eta_{V} \rho_{U}-\eta_{U} \rho_{V}\right) \\
& +\eta_{I}\left(\frac{\partial \eta_{V}}{\partial x} \rho_{U}+\eta_{V} \frac{\partial \rho_{U}}{\partial x}-\frac{\partial \eta_{U}}{\partial x} \rho_{V}-\eta_{U} \frac{\partial \rho_{V}}{\partial x}\right) \\
\frac{\partial T_{3}}{\partial x}= & 2 \eta_{I} \frac{\partial \eta_{I}}{\partial x} \eta_{U}+\eta_{I}^{2} \frac{\partial \eta_{U}}{\partial x}+\frac{\partial \eta_{I}}{\partial x}\left(\eta_{Q} \rho_{V}-\eta_{V} \rho_{Q}\right) \\
& +\eta_{I}\left(\frac{\partial \eta_{Q}}{\partial x} \rho_{V}+\eta_{Q} \frac{\partial \rho_{V}}{\partial x}-\frac{\partial \eta_{V}}{\partial x} \rho_{Q}-\eta_{V} \frac{\partial \rho_{Q}}{\partial x}\right) \\
\frac{\partial T_{4}}{\partial x}= & 2 \eta_{I} \frac{\partial \eta_{I}}{\partial x} \eta_{V}+\eta_{I}^{2} \frac{\partial \eta_{V}}{\partial x}+\frac{\partial \eta_{I}}{\partial x}\left(\eta_{U} \rho_{Q}-\eta_{Q} \rho_{U}\right) \\
& +\eta_{I}\left(\frac{\partial \eta_{U}}{\partial x} \rho_{Q}+\eta_{U} \frac{\partial \rho_{Q}}{\partial x}-\frac{\partial \eta_{Q}}{\partial x} \rho_{U}-\eta_{Q} \frac{\partial \rho_{U}}{\partial x}\right), \\
\frac{\partial T_{5}}{\partial x}= & 2\left(\eta_{I} \frac{\partial \eta_{I}}{\partial x}-\eta_{Q} \frac{\partial \eta_{Q}}{\partial x}-\eta_{U} \frac{\partial \eta_{U}}{\partial x}-\eta_{V} \frac{\partial \eta_{V}}{\partial x}+\rho_{Q} \frac{\partial \rho_{Q}}{\partial x}\right. \\
& \left.+\rho_{U} \frac{\partial \rho_{U}}{\partial x}+\rho_{V} \frac{\partial \rho_{V}}{\partial x}\right) .
\end{aligned}
$$

The derivatives with respect to $\eta_{0}$ can be easily calculated from Eq. (A.2)

$$
\begin{aligned}
\frac{\partial \eta_{I}}{\partial \eta_{0}} & =\frac{\left(\eta_{I}-1\right)}{\eta_{0}}, \\
\frac{\partial \eta_{Q, U, V}}{\partial \eta_{0}} & =\frac{\eta_{Q, U, V}}{\eta_{0}}, \\
\frac{\partial \rho_{Q, U, V}}{\partial \eta_{0}} & =\frac{\rho_{Q, U, V}}{\eta_{0}} .
\end{aligned}
$$

The derivatives with respect to $\gamma$ and $\psi$ are

$\frac{\partial \eta_{I}}{\partial \chi}=0, \quad \frac{\partial \eta_{V}}{\partial \chi}=0, \quad \frac{\partial \rho_{V}}{\partial \chi}=0$,

$\frac{\partial \eta_{Q}}{\partial \chi}=-2 \eta_{Q} \tan 2 \chi$ 
D. Orozco Suárez and J. C. del Toro Iniesta: The usefulness of analytic response functions, Online Material p 3

$\frac{\partial \eta_{U}}{\partial \chi}=2 \eta_{U} \cot 2 \chi$

$\frac{\partial \rho_{Q}}{\partial \chi}=-2 \rho_{Q} \tan 2 \chi$

$\frac{\partial \rho_{U}}{\partial \chi}=2 \rho_{U} \cot 2 \chi$

$\frac{\partial \eta_{I}}{\partial \gamma}=\frac{\eta_{0}}{2}\left[\phi_{p}-\frac{\phi_{b}+\phi_{r}}{2}\right] \sin 2 \gamma$

$\frac{\partial \eta_{Q}}{\partial \gamma}=\frac{\eta_{0}}{2}\left[\phi_{p}-\frac{\phi_{b}+\phi_{r}}{2}\right] \sin 2 \gamma \cos 2 \chi$,

$\frac{\partial \eta_{U}}{\partial \gamma}=\frac{\eta_{0}}{2}\left[\phi_{p}-\frac{\phi_{b}+\phi_{r}}{2}\right] \sin 2 \gamma \sin 2 \chi$

$\frac{\partial \eta_{V}}{\partial \gamma}=-\eta_{V} \tan \gamma$

$\frac{\partial \rho_{Q}}{\partial \gamma}=\frac{\eta_{0}}{2}\left[\psi_{p}-\frac{\psi_{b}+\psi_{r}}{2}\right] \sin 2 \gamma \cos 2 \chi$,

$\frac{\partial \rho_{U}}{\partial \gamma}=\frac{\eta_{0}}{2}\left[\psi_{p}-\frac{\psi_{b}+\psi_{r}}{2}\right] \sin 2 \gamma \sin 2 \chi$,

$\frac{\partial \rho_{V}}{\partial \gamma}=-\rho_{V} \tan \gamma$

The derivatives with respect to the other parameters imply the derivatives of the absorption and dispersion profiles and these lead us to obtain the derivatives of the Voigt and Voigt-Faraday functions (as defined by Landi degl'Innocenti 1976):

$$
\begin{aligned}
\frac{\partial \phi_{j}}{\partial x} & =\sum_{M_{1}-M_{\mathrm{u}}=j} S_{M_{\mathrm{l}} M_{\mathrm{u}}, j} \frac{\partial H(a, v)}{\partial x}, \\
\frac{\partial \psi_{j}}{\partial x} & =2 \sum_{M_{1}-M_{\mathrm{u}}=j} S_{M_{\mathrm{l}} M_{\mathrm{u}}, j} \frac{\partial F(a, v)}{\partial x} .
\end{aligned}
$$

By using the chain rule and the derivatives of $H(a, v)$ and $F(a, v)$ with to respect $a$ and $v$,

$$
\begin{aligned}
& \frac{\partial H(a, v)}{\partial a}=-2 \frac{\partial F(a, v)}{\partial v}, \\
& \frac{\partial F(a, v)}{\partial a}=\frac{1}{2} \frac{\partial H(a, v)}{\partial v}, \\
& \frac{\partial H(a, v)}{\partial v}=4 a F(a, v)-2 v H(a, v), \\
& \frac{\partial F(a, v)}{\partial v}=\frac{1}{\sqrt{\pi}}-a H(a, v)-2 v F(a, v),
\end{aligned}
$$

we find

$$
\begin{aligned}
& \frac{\partial H(a, v), F(a, v)}{\partial B}=\frac{\partial H(a, v), F(a, v)}{\partial v} \frac{\Delta \lambda_{i_{j}}}{\Delta \lambda_{\mathrm{D}}} \frac{1}{B}, \\
& \frac{\partial H(a, v), F(a, v)}{\partial v_{\mathrm{LOS}}}=\frac{\partial H(a, v), F(a, v)}{\partial v} \frac{-\lambda_{0}}{c \Delta \lambda_{\mathrm{D}}}, \\
& \frac{\partial H(a, v), F(a, v)}{\partial \Delta \lambda_{\mathrm{D}}}=\frac{\partial H(a, v), F(a, v)}{\partial v} \frac{-v}{\Delta \lambda_{\mathrm{D}}} .
\end{aligned}
$$

